\title{
The economic burden of constipation therapy
}

Anamaria Albu ${ }^{1}$, Andreea Farcas², Liliana David', Dan L. Dumitrascu ${ }^{1}$

1) $2^{\text {nd }}$ Department of Internal Medicine, Iuliu Hatieganu University of Medicine and Pharmacy, ClujNapoca, Romania

2) Drug Information Research Center, Iuliu Hatieganu University of Medicine and Pharmacy, ClujNapoca, Romania
DOI: $10.15386 / \mathrm{mpr}-1222$

Manuscript received: 29.10.2018

Received in revised form: 28.12.2018

Accepted: 17.01.2019

Address for correspondence:

afarcas@umfcluj.ro

\begin{abstract}
Background and aim. Constipation is a widespread condition that requires adequate therapy. Given the large use of laxatives, it is important to know the burden of the treatment of this condition on the healthcare budget.
\end{abstract}

Methods. We collected information on the number of therapeutic units (boxes) of five frequently used laxatives delivered by prescription or over the counter in 30 pharmacies randomly selected in two adjacent counties from Romania for two consecutive years. We calculated also the cost value of the laxatives delivered.

Results. The total cost of laxatives in the group of pharmacies investigated was on average 62,500 euros per year. We extrapolated that the laxative consumption in Romania should be over 15 million Euro.

Conclusions. To our knowledge this is the first study on the burden of constipation in this country. Although therapeutic units of laxatives are not expensive, the wide use of these drugs represents an economic burden for the patient.

Keywords: constipation, drug, health economics, pharmaeconomics

\section{Introduction}

Constipation is one of the most widespread clinical conditions, with increasing prevalence in the last century and also with ageing [1]. Its definition may rely on the patient's and on physician's perception: hard stools, rare defecation, and difficult defecation. With the advent of the Bristol scale [2], now largely used and validated in many countries [3], constipation is better defined by stool form.

Constipation is a major concern for the health budgets and may cause an important socio-economic burden [4]. It is not easy to estimate the cost of constipation seen only from the perspective of the cost of laxative therapies. Most patients prefer dietary changes, self medication, or alternative therapies, before entering the official healthcare providing system. Therefore we can only assess "the visible part of the iceberg", i.e. drugs for which records of delivery exist.

Looking to the future, it is important to consider the ageing of the general population and the estimated increasing costs for treating constipation. New drugs came out recently [5] on the market and their price is expected to increase the cost of the management of constipation [6].

\section{Background and aim}

Given that constipation is a serious burden both for the health and the patients' budget, because of its high prevalence and of many chronic forms of presentation [7], and that data on the cost of constipation in our country Romania are missing, we aimed to estimate the cost of constipation therapy in this country. We looked therefore for the socio-economic impact of constipation in our area, by collecting and analyzing data on the delivery in pharmacies of drugs for constipation.

\section{Methods \\ Protocol}

This investigation was carried out in pharmacies from two Romanian counties: Cluj and Alba. These are representative and neighboring counties in Romania, having a total population of about one million: (690 000 Cluj, $70 \%$ in urban area; 342000 in Alba, $60 \%$ in urban area). We extrapolated this information to the whole country. The cost of drugs are homogenous in Romania, thus the price of drugs in these counties are similar to those from whole country (official statistical data).

\section{Pharmaceutical units}

We made a thorough analysis of 
the pharmaceutical units available in two counties. During our study period there were 116 pharmacies in Alba county, 74 having contract with the National Insurance House (data from the Alba County Health Insurance House) and 299 Pharmacies in Cluj County (listed by the Cluj College of Pharmacists) 135 of them having reimbursement contracts with the National Health Insurance House (data collected from the Cluj County Health Insurance House).

We collected data in 30 randomly selected pharmacies for 2 consecutive years (2013 and 2014). From these, 18 were in Alba county (12 in urban and 6 rural area) and 12 pharmacies were in the neighbor county Cluj (10 urban, 2 rural).

\section{Data collected}

We asked and got information in all pharmacies (100\% response rate) on the number of units and cost for 5 laxative drugs with following commercial names: Dulcolax $\AA$, Sennalax $\AA$, Ciocolax $\AA$, Duphalac $\AA$ and glycerin suppositories. Dulcolax ${ }^{\circledR}$ is a contact laxative containing 5 mg bisacodyl; Sennalax ${ }^{\circledR}$ is an extract of Senna acutifolia; Ciocolax ${ }^{\circledR}$ is phenolphtaleine with a more palatable cacao ingredient; Duphalac ${ }^{\circledR}$ is lactulose; Glycerin suppositories are also frequently used for constipation, including the administration to children.

All pharmacies reported the number of units (boxes) delivered and its cost for each year. In addition we collected information on the number of prescriptions vs. "over the counter" (OTC) use for Duphalac $\AA$. For glycerin suppositories we received data for both pediatric and adult use.

\section{Statistics}

Statistic calculations were carried out on a commercially available software and included descriptive statistics and t-test for comparisons. Significance level was set at $\mathrm{p}<0.05$.

\section{Results}

\section{Therapeutic units released}

The number of laxative units released in the 30 pharmacies for each product per year and per each county is presented in Table I.

One can see that suppositories with glycerin $\AA$, Sennalax ${ }^{\circledR}$ and Dulcolax ${ }^{\circledR}$ have the largest use. These data reflect both medical prescription preferences as well as patients' preferences. These are relatively cheap drugs but the extensive use creates an economic burden on the patient or on the healthcare budget if reimbursed.
Regarding the pediatric use of glycerin suppositories $\AA$, it represents 5684 units, i.e. $32 \%$ from the total use of this drug.

Duphalac ${ }^{\circledR}$ was delivered either by prescription or as OTC. 2722 units of Duphalac ${ }^{\circledR}$ were sold over the counter compared to Duphalac $\AA$ sold on prescription (1105 units). It means that the OTC use of this lactulose commercial product is almost 3 times more frequent than on prescription.

\section{The cost of five main laxatives}

The prices were also analyzed. Prices are homogenous across the county, but small differences may occur between pharmacies. In general, pharmaceutical chains and pharmaceutical units in rural areas sell usually less expensive drugs compared to individual pharmacies in urban areas. We collected the money value from the sales records of the pharmacy units participating in this study.

In Table II we display the cost in RON for the main laxatives analyzed in this study $(1 \mathrm{Euro}=4.5 \mathrm{RON}$ at the time of the study).

Table II. The total cost of the main laxatives.

\begin{tabular}{l|c|}
\hline Drug & Cost (RON) \\
\hline Glycerine supp. & 133612 \\
Duphalac & 140989 \\
Ciocolax & 13065 \\
Sennalax & 112310 \\
Dulcolax & 160986
\end{tabular}

The total cost of the constipation therapy with laxatives in these 2 counties for 2 years was: 560962 RON. This represents a total of 125000 Euro for two years. There was a slight increase in the consumption of laxatives in the second year compared with the previous year. The increase was not significant and was not considered for analysis. Thus the annual five laxatives cost was about 62,500 EUR. Most of this cost $(57,800$ EUR) is supported by the patient, as most of these laxatives were OTCs drugs, except some Duphalac $\AA$ reimbursed prescriptions.

There was a higher consumption of the five laxatives in the Cluj county: 322,416 RON than in Alba county (which has a smaller population): $241,358 \mathrm{RON}$.

We emphasize that these data represent information from sales recordings and largely underscores the real life use and costs of laxatives.

Table I. Total number of released laxative units.

\begin{tabular}{|l|c|c|c|c|c|}
\hline Laxatives & RU AB 2013 & RU CJ 2013 & RU AB 2014 & RU CJ 2014 & Total RU \\
\hline Dulcolax ${ }^{\circledR}$ & 3313 & 3793 & 3477 & 5126 & 15709 \\
Sennalax ${ }^{\circledR}$ & 1770 & 4086 & 1864 & 5825 & 13545 \\
Ciocolax ${ }^{\circledR}$ & 323 & 253 & 332 & 451 & 1359 \\
Duphalac ${ }^{\circledR}$ & 955 & 791 & 924 & 1207 & 3877 \\
Glycerine Suppositories & 3080 & 4807 & 3734 & 6283 & 17904
\end{tabular}

RU - Released Units; AB - Alba County; CJ - Cluj County 


\section{Extrapolations}

From these data we attempted an extrapolation of the total cost supported by the patient for the five most commonly used laxatives for the whole country (19 million inhabitants). We analyzed 30 pharmacies from a total of 415 pharmacies in both counties. It can be assumed that the estimated total cost for one year for the five laxatives in the two counties is given by a multiplication by 13.8 . This gives an approximated cost of 797,640 euros. Considering this cost for an area with one million inhabitants, the total estimated cost for the five most commonly prescribed laxatives in Romania should be around 15,155,160 EUR. According to this estimation each Romanian spends about 0.80 EUR for the five most important laxatives. But given the large volume of sales, the costs run high. Due to the low number of reimbursed prescriptions, the cost for the healthcare budget is negligible.

These prices are however low compared with more industrialized countries.

\section{Discussion}

To our knowledge, this is the first medical report on the burden of constipation therapy in Romania as reflected by the cost of used laxatives. In order to estimate the patients' budget allocated to laxatives we used a significant sample of pharmacies in two neighbored counties in central western part of Romania and looked for two consecutive years to the use of the five main laxatives.

We observed that in this area the recorded consumption of laxatives on prescription and over the counter was of 62,500 EUR yearly, out of which 57,800 EUR represents the patients' own expenses. Considering for the whole Romanian population of 19 millions, it gives an approximated total of 15,155,160 EUR.

But the burden of constipation is not limited to the use of laxatives $[7,8]$. We have to consider that constipation requires beside laxatives also other drugs, for instance antidepressants [9]. Beside this, there is the cost of investigations, emergency department presentation or chronic hospitalization, and even treatment failure associated costs, which all impact the healthcare budget. In the USA between 2006-2011 the cost for emergency presentation because of constipation almost doubled [10]. On a longer time span survey of 13 years an important increase of the cost for constipation was also observed in USA, related to hospitalization [11]. In another paper, analyzing the treatment failure for chronic constipation and irritable bowel syndrome with predominant constipation, it has been observed that failure increased significantly the management cost of these conditions [12].

There was an important amount of money spent on different categories of these main laxative drugs in the 30 pharmacies taken as index for laxative consumption. However, the Romanian patients' expenditure for the treatment of constipation is much lower than in US for example, where annual out-of-pocket expenses averaged $390 \$$ for constipation [7]. In one Swedish study, the average annual cost per patient with constipation was $60 €$, reflecting prescription drugs [13].

A recent systematic review of cost effectiveness of different therapies for chronic constipation concluded that all are cost-effective except some alternative practices [14]. One can consider that treating constipation, not only with laxatives, is cost-effective. Cases with failure to laxatives require more expensive interventions with more recent drugs.

Our study has however several limitations evolving from the regional origin of the data and the extrapolation of the cost. Although the information collected from the 30 pharmaceutical units involved in the study is accurate, the extrapolation in respect to the total number of pharmacies in the two counties where the study was conducted and to the general Romanian population represents approximations. However it is important to have such data in order to assess the economical burden of constipation in this country. Moreover, part of prescription Duphalac could have been for patients with liver cirrhosis (encephalopathy prevention or treatment) and not for constipation. Based on our data, more systematic investigations on this topic can be further performed.

\section{Conclusions}

The total estimated cost per year of laxatives used by Romanian patients is over 15 million EUR as calculated from the records of released pharmaceutical units. Even in countries with medium economical development, constipation represents an important toll for the patients' budget.

\section{References}

1. Forootan M, Bagheri N, Darvishi M. Chronic constipation: A review of literature. Medicine (Baltimore). 2018;97(20):e10631.

2. Lewis SJ, Heaton KW. Stool form scale as a useful guide to intestinal transit time. Scand J Gastroenterol. 1997;32:920-924.

3. Chira A, Dumitrascu DL. Validation of the Bristol Stool Form Scale into Romanian.J Gastrointestin Liver Dis. 2015;24:539540 .

4. Leung L, Riutta T, Kotecha J, Rosser W. Chronic constipation: an evidence-based review. J Am Board Fam Med. 2011;24:436451.

5. Bharucha AE, Wouters MM, Tack J. Existing and emerging therapies for managing constipation and diarrhea. Curr Opin Pharmacol. 2017;37:158-166.

6. Sanchez MI, Bercik P. Epidemiology and burden of chronic constipation. Can J Gastroenterol. 2011;25 (Suppl B):11B$15 \mathrm{~B}$.

7. Nyrop KA, Palsson OS, Levy RL, Von Korff M, Feld AD, Turner MJ, et al. Costs of health care for irritable bowel syndrome, chronic constipation, functional diarrhoea and functional abdominal pain. Aliment Pharmacol Ther. 
2007;26:237-248.

8. Dennison C, Prasad M, Lloyd A, Bhattacharyya SK, Dhawan $\mathrm{R}$, Coyne $\mathrm{K}$. The health-related quality of life and economic burden of constipation. Pharmacoeconomics. 2005;23:461476.

9. Dumitraşcu DL, Acalovschi M, Pascu O. Hostility in patients with chronic constipation. Rom J Intern Med. 1998;36:239243.

10. Sommers T, Corban C, Sengupta N, Jones M, Cheng $\mathrm{V}$, Bollom A, et al. Emergency department burden of constipation in the United States from 2006 to 2011. Am J Gastroenterol. 2015;110:572-579.

11. Sethi S, Mikami S, Leclair J, Park R, Jones M, Wadhwa V, et al. Inpatient burden of constipation in the United States: an analysis of national trends in the United States from 1997 to 2010. Am J Gastroenterol. 2014;109:250-256.

12. Guerin A, Carson RT, Lewis B, Yin D, Kaminsky M, Wu E. The economic burden of treatment failure amongst patients with irritable bowel syndrome with constipation or chronic constipation: a retrospective analysis of a Medicaid population. J Med Econ. 2014;17:577-586.

13. Bruce Wirta S, Hodgkins P, Joseph A. Economic burden associated with chronic constipation in Sweden: a retrospective cohort study. Clinicoecon Outcomes Res. 2014;6:369-379.

14. Han D, Iragorri N, Clement F, Lorenzetti D, Spackman E. Cost Effectiveness of Treatments for Chronic Constipation: A Systematic Review. Pharmacoeconomics. 2018;36:435-449. 